\title{
Macroscopic Expressions of Molecular Adiabatic Compressibility of Methyl and Ethyl Caprate under High Pressure and High Temperature
}

\author{
Fuxi Shi, ${ }^{1}$ Qin Zhang, ${ }^{2}$ Jun Chen, ${ }^{1}$ and Hamid Reza Karimi ${ }^{4}$ \\ ${ }^{1}$ College of Mechanical and Electronic Engineering, Northwest A\&F University, No. 22, Xinong Road, Yangling, Xian, \\ Shaanxi 712100, China \\ ${ }^{2}$ Department of Biological Systems Engineering, Washington State University, Room 10524106 N Bunn Road, Prosser, WA, USA \\ ${ }^{4}$ Department of Engineering, Faculty of Engineering and Science, University of Agder, 4898 Grimstad, Norway
}

Correspondence should be addressed to Jun Chen; chenjun_jdxy@nwsuaf.edu.cn

Received 18 November 2013; Accepted 18 December 2013; Published 14 January 2014

Academic Editor: Ming Liu

Copyright (C) 2014 Fuxi Shi et al. This is an open access article distributed under the Creative Commons Attribution License, which permits unrestricted use, distribution, and reproduction in any medium, provided the original work is properly cited.

\begin{abstract}
The molecular compressibility, which is a macroscopic quantity to reveal the microcompressibility by additivity of molecular constitutions, is considered as a fixed value for specific organic liquids. In this study, we introduced two calculated expressions of molecular adiabatic compressibility to demonstrate its pressure and temperature dependency. The first one was developed from Wada's constant expression based on experimental data of density and sound velocity. Secondly, by introducing the 2D fitting expressions and their partial derivative of pressure and temperature, molecular compressibility dependency was analyzed further, and a 3D fitting expression was obtained from the calculated data of the first one. The third was derived with introducing the pressure and temperature correction factors based on analogy to Lennard-Jones potential function and energy equipartition theorem. In wide range of temperatures $(293<\mathrm{T} / \mathrm{K}<393)$ and pressures $(0.1<\mathrm{P} / \mathrm{MPa}<210)$, which represent the typical values used in dynamic injection process for diesel engines, the calculated results consistency of three formulas demonstrated their effectiveness with the maximum $0.5384 \%$ OARD; meanwhile, the dependency on pressure and temperature of molecular compressibility was certified.
\end{abstract}

\section{Introduction}

In modern diesel engine, the injection pressure of fuel spray system continuously was elevated to achieve better atomization, combustion, and emission effect and even reach $200 \mathrm{MPa}$ or more, which increase vastly the dynamics sensibility of fuel compressibility. The dynamic delivery process, which was carried out under rapid variation of pressure and temperature, was strongly affected by the compressibility of fuel and its derivative properties relative to pressure and temperature. For example, density influences the conversion of volume flow rate into mass flow rate [1], whereas the compressibility (the reciprocal of bulk modulus) acts on the fuel injection timing $[2,3]$. Therefore, the adaptation of injection systems to biodiesels requires an accurate knowledge of the volumetric properties of biodiesel components over a wide range of pressure and temperature $[4,5]$; especially the compressibility and sound velocity values were indispensable parameters for system modeling and experimental injection rate determination [6].

In order to clarify the connection between the microscopic chemical structure of organic liquids and the compressibility from investigation of sound velocity and density, many theoretical analyses and derivations have been performed to determine these properties. Rao [7] has pointed out the product of density and 7th root of isothermal compressibility $k_{T}, \rho k_{T}^{1 / 7}$ was highly independent of temperature and pressure. Further, molecular adiabatic compressibility, $k_{m}=M_{w} / \rho k_{s}^{1 / 7}$, which was proposed by Wada [8] and was calculated by summing bond increments of molecular components, was used to express the microcompressibility with 
a constant molar volume for many organic liquids. Schaaffs $[9,10]$, who firstly derived a quantitative expression to create connection between sound velocity and chemical structure of organic compounds, pointed out the properties related to sound velocity with the atomic additive property [11]. As the eigenvalues of the molecular spacing and molecular intermolecular forces, molecular compressibility was regarded as a constant.

Nowadays, molecular compressibility was used to predict the speed of sound in some research [12-14]; a negative temperature correction coefficient is used to modify the influence of temperature on molecular compressibility; it is an effective solution to decrease prediction deviation when the numerical accuracy of molecular compressibility was required. Freitas et al. [13] predicted speed of sound of FAMEs by Wada's group contribution method, but the ARD of predicted results shows regularly downward trend with elevated temperature. Later Daridon et al. [12] divided the FA esters molecule into five bigger functional groups to contribute to the molecular compressibility; meanwhile molecular compressibility was demonstrated with slight temperature dependency. These investigations provide a quantitative connection between macroproperties and molecular microstructure, but the literature on the compressibility for FA esters and biodiesels is still very scant relative to its species; most data are available only at atmospheric pressure and in a narrow range of temperature and could not match the fuel working conditions in injection system. That restricted the overall cognition on molecular compressibility. Recently, more studies $[15,16]$, which focused on sound velocity and density under high pressure and high temperature conditions and the modeling of these properties, gave a possibility to comprehensively understand on molecular adiabatic compressibility in $P-T$ domain.

In this paper, the calculated value of molecular compressibility was extrapolated to close working conditions of fuel injection system [17] based on [15] data. By data fitting, its dependence of pressure and temperature was formulated and analyzed at two dimensions and three dimensions. As the eigenvalues of the molecular spacing and molecular intermolecular forces, molecular compressibility was connected to molecular potential energy and thermal kinetic energy by developing a new correction formula. The consistency of calculated results derived from different principles verified effectiveness of these formulas and confirmed the dependency of molecular compressibility on pressure and temperature.

\section{The Theory of the Molecular Compressibility}

Concept of molecular compressibility was derived from the summary of the experimental data of organic liquids [8]. Wada has pointed out that if $\rho k_{S}^{1 / 7}$ is independent of pressure, the rate of $1 / k_{s} \cdot \partial k_{s} / \partial p$ to $1 / \rho \cdot \partial \rho / \partial p$ was expected to be a constant $n$, which was 7.3 in many organic liquids. So $\rho k_{s}^{1 / 7}$ was used as a characteristic function of organic liquids; thus molecular adiabatic compressibility $M_{w} / \rho k_{s}^{1 / 7}$ is expressed as additivity of molecular constitution and shows an approximate fixed value for an organic liquid. But this application will bring two aspects problems. Firstly, $n=7$ is just an approximate value based on experimental data; this application is short of theoretical basis and must have certain error under a large range of pressure and temperature. Secondly, the difference of molecular configuration and size responding to pressure and temperature should be distinct; although Wada listed about one hundred calculated values of molecular compressibility to show additive property of bond increments, still seven or more organic liquids have appreciable discrepancies between experimental and calculated values, and the experimental conditions of data acquisition were not shown clearly.

In Wada's derivation, molecular adiabatic compressibility was expressed as

$$
k_{m}=\frac{M_{w}}{\rho k_{s}^{1 / 7}}=\frac{V_{0}}{k_{s 0}^{1 / 7}}=\text { Wada's Const., }
$$

where $M_{w}, V_{0}, k_{s 0}$ are the molecular weight, the mole volume at close packed state and the compressibility of molecule itself, respectively, and shown as:

$$
\begin{gathered}
\frac{M_{w}}{\rho}=V_{0}+v=V_{0}(1+k), \\
k_{s}=k_{s 0}(1+k)^{7},
\end{gathered}
$$

where $v, k$ are the free volume per mole and fractional free volume, respectively. For different organic liquid, $V_{0}$ as the molar volume of close packed state is fixed value, $k_{s 0}^{1 / 7}$ also are shown as an almost fixed calculated value; thus Wada concluded $k_{m}$ was approximately constant. But there exists an unreasonable assumption that $V_{0}$ is unlikely to reach the close packed state at liquid state; hence $V_{0}$ compressibility is variational under dynamic high pressure and high temperature. Furthermore 7 th power of $(1+k)$ in formula (2) is incorrect to express the linear superposition of compressibility between free volume and molecular size. So we can draw a conclusion that the consistent $k_{s 0}^{1 / 7}$ value is derived from tiny error with 7 th root of $k_{s 0}$, so the formula (1) is not strictly correct and approximate algorithm under some special conditions.

In addition, the formula (1) can further be developed according to the definition of adiabatic compressibility $k_{s}$ as follows:

$$
\begin{aligned}
k_{m} & =\frac{M_{w}}{\rho k_{s}^{1 / 7}}=\frac{M_{w}}{\rho(-(1 / V)(\partial V / \partial P))^{1 / 7}} \\
& =\frac{M_{w}}{\rho((1 / \rho)(\partial \rho / \partial P))^{1 / 7}}=\frac{M_{w}}{\rho^{6 / 7}}\left(\frac{\partial P}{\partial \rho}\right)^{1 / 7} .
\end{aligned}
$$

So molecular adiabatic compressibility is supposed to be a function of density with pressure, and the influence of the temperature is implied in density. 
TABLE 1: Group contribution values for molecular compressibility estimation at atmospheric pressure [12].

\begin{tabular}{lcccc}
\hline & $-\mathrm{CH}_{3}$ & $-\mathrm{CH}_{2}-$ & $-\mathrm{CH}=\mathrm{CH}-$ & $\mathrm{CH}_{3} \mathrm{COO}_{-}$ \\
\hline$K_{m} \times 10^{3}$ & 0.5097 & 0.35196 & 0.59074 & 1.05856 \\
$T_{0}(\mathrm{~K})$ & & & 298.15 & \\
$\chi \times 10^{3}$ & & & 0.034852 & \\
\hline
\end{tabular}

\section{The Characteristic Analysis of the Molecular Compressibility}

For revealing similar law of the properties, many density and sound velocity measurements for FAMEs and FAEEs were performed and predictive methodologies were proposed to determine the sound velocity based on ultrasonic technique. Tat et al. and Tat and van Gerben [21, 22] obtained density and sound velocity of $16 \mathrm{FA}$ esters in 20 to $100^{\circ} \mathrm{C}$ and 0.1 to $32.5 \mathrm{MPa}$ and achieved only one polynomial function, which is second order in temperature and first order in pressure with different fitting coefficients, to predict density, sound velocity and isentropic bulk modulus whose dependency on temperature and pressure indicates similitude principle of change for different FA esters, (4) was used to convert the isentropic bulk modulus $\beta_{s}$ to isentropic compressibility:

$$
k_{s}=\frac{1}{\beta_{s}}=\frac{1}{\rho c^{2}} .
$$

Daridon et al. [12] measured sound velocity and density of seven FA esters (including ethyl and methyl, saturated and unsaturated) by using a pulse echo technique operating with $3 \mathrm{MHz}$ at atmospheric pressure in $283.15 \sim 373.15 \mathrm{~K}$ range. The molecular compressibility, which was calculated by Wada's group contribution method, was almost a constant for specific FA ester. the five basic microscopic radicals $\left(\mathrm{CH}_{3} \mathrm{COO}-\right.$, $-\mathrm{CH}_{2} \mathrm{COO}-, \mathrm{CH}_{3}-,-\mathrm{CH}_{2}-,-\mathrm{CH}=\mathrm{CH}-$ ), which contributed to molecular compressibility of a FA ester according to additivity and were listed in Table 1, had a fixed value, respectively, and the predicted results for sound velocity (using (6)) showed a high prediction accuracy. This study showed that the molecular compressibility is related to molecular specific structure, and the negative temperature correction coefficient in (5) presented temperature dependence of molecular compressibility:

$$
\begin{gathered}
k_{m}(T)=\sum_{j=1}^{n_{G}} N_{j} k_{m j}(1-\chi(T-298.15)), \\
c=\rho^{3}\left(\frac{k_{m}}{M_{w}}\right)^{7 / 2},
\end{gathered}
$$

where $k_{m}, k_{s}, M_{w}, \rho, c$ are molecular compressibility, isentropic compressibility, the molecular weight density, and sound velocity, respectively, $k_{m j}$ is the contribution of the group type- $j$ to $k_{m}$ which occurs $N_{j}$ times in the given molecule, and $\chi$ is a constant parameter used to take into account the influence of temperature.

Freitas et al. [13] also used Wada's model to predict the sound velocity of the fatty acid esters and biodiesels with
OARDs of $0.25 \%$ and $0.45 \%$. But these predicted results of high precision, which show evidence of effectiveness of Wada's model, are just achieved at atmospheric pressure within a small temperature range. Latter Freitas et al. [14] developed Wada's model to predict the sound velocity of FA esters at high pressures up to $35 \mathrm{MPa}$ and pointed out that the pressure dependency of the sound velocity is approximately linear relation in the pressure range considered, in consideration of the nonlinear of $\rho^{3}$ under variable pressure; thus the molecular compressibility must not be a constant. So it is necessary to extrapolate the value and tendency of molecular compressibility in high pressure and high temperature.

3.1. Characteristic of Molecular Isentropic Compressibility in High Pressure and High Temperature. Ndiaye et al. [15] measured sound velocity of methyl caprate and ethyl caprate at pressures up to $210 \mathrm{MPa}$ in the temperature range 283.15 to $403.15 \mathrm{~K}$. In addition, density was measured in 0.1 to $100 \mathrm{MPa}$ and 293.15 to $393.15 \mathrm{~K}$, by using a modification of Davis and Gordon's procedure [23]; the method [24] rested on the Newton-Laplace relationships; then density was extrapolated up to $210 \mathrm{MPa}$ from sound velocity data (7) with an absolute average deviation of $0.07 \%$ up to $210 \mathrm{MPa}$. the isentropic and isothermal compressibilities also were presented in the $P-T$ domain:

$$
\rho(P, T)=\rho\left(P_{\mathrm{ref}}, T\right)+\int_{P_{\mathrm{ref}}}^{P} \frac{1}{c^{2}} d P+T \int_{P_{\mathrm{ref}}}^{P} \frac{\alpha_{P}^{2}}{C_{p}} d P,
$$

where $C_{p}, \alpha_{p}$ represent the isobaric heat capacity and the isobaric thermal expansion, respectively, and $P_{\text {ref }}$ is a reference pressure where density is known.

In order to directly obtain molecular isentropic compressibility $k_{m}$ from experimental data, we expressed $k_{m}$ as a function of density and sound velocity, shown as (8).

$$
k_{m}=M_{w}\left(\frac{c}{\rho^{3}}\right)^{2 / 7} .
$$

Based on [15] data that sound velocity was obtained from experimental and density was obtained from calculated value by (7), $k_{m}$ was calculated in 0.1 to $210 \mathrm{MPa}$ and 303.15 to $383.15 \mathrm{~K}$ and the fitting results were presented with isothermal characteristics for methyl caprate and ethyl caprate in Figures 1 and 2.

A one-order rational fitting relation to pressure was expressed as (9), where $P$ is pressure, other parameters $A_{1}$, $A_{2}, A_{3}$ are equation coefficients, as listed in Table 2:

$$
k_{m}(P)=\frac{A_{1} P+A_{2}}{P+A_{3}}=a+\frac{b}{P+c} .
$$


TABLE 2: Fitting coefficients for (9) along various isothermals.

\begin{tabular}{|c|c|c|c|c|c|c|}
\hline \multirow{2}{*}{$\begin{array}{l}\text { FA ester } \\
\text { Temperature }\end{array}$} & \multicolumn{3}{|c|}{ Methyl caprate } & \multicolumn{3}{|c|}{ Ethyl caprate } \\
\hline & $303.15 \mathrm{~K}$ & $343.15 \mathrm{~K}$ & $383.15 \mathrm{~K}$ & $303.15 \mathrm{~K}$ & $343.15 \mathrm{~K}$ & $383.15 \mathrm{~K}$ \\
\hline \multicolumn{7}{|l|}{ Coefficients } \\
\hline$A_{1}$ & 4.597 & 4.612 & 4.632 & 4.985 & 4.992 & 5.004 \\
\hline$A_{2}$ & 480.8 & 374.7 & 291.4 & 547.2 & 423.4 & 303 \\
\hline$A_{3}$ & 109.7 & 85.55 & 66.58 & 115.5 & 89.39 & 63.96 \\
\hline \multicolumn{7}{|l|}{ Goodness of fit } \\
\hline SSE & $2.25 E-06$ & $2.45 E-06$ & $8.37 E-06$ & $1.48 E-06$ & $5.76 E-06$ & $8.34 E-06$ \\
\hline$R$-square & 0.9999 & 0.9999 & 0.9998 & 1 & 0.9999 & 0.9999 \\
\hline Adjusted $R$-square & 0.9999 & 0.9999 & 0.9998 & 1 & 0.9999 & 0.9998 \\
\hline RMSE & 0.000375 & 0.000391 & 0.000723 & 0.000304 & 0.0006 & 0.000722 \\
\hline
\end{tabular}

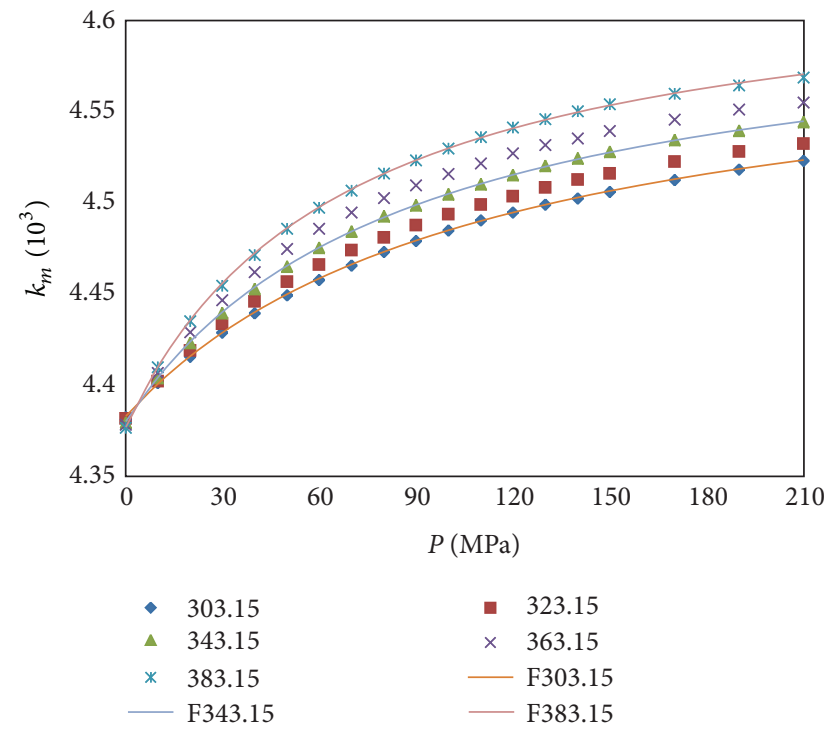

FigURE 1: Molecular compressibility of methyl caprate (MeC10:0) as a function of pressure along various isothermal (the lines are fitting results; the dots are calculated values).

The rational relation (9) was achieved with satisfying goodness of fit, where the minimum $R$-square value is 0.9998 in all temperature domains. We may safely draw the conclusion that molecular compressibility is dependent on pressure for ethyl and methyl caprate; this dependence on pressure was revealed sufficiently with elevated pressure In Figures 1 and 2.

Accordingly, the calculated results were presented with isobaric characteristics for methyl caprate and ethyl caprate in Figures 3 and 4, and a two-order polynomial fitting in temperature domain was expressed as

$$
k_{m}(T)=B_{1} T^{2}+B_{2} T+B_{3}=a(T+b)^{2}+c,
$$

where $T$ is temperature and other parameters $B_{1}, B_{2}, B_{3}$ are equation coefficients, as listed in Table 3.

In Figures 3 and 4, a two-order polynomial (10) was achieved with the minimum $R$-square value being 0.9987 in all pressure domains. At atmospheric pressure (0.1 MPa isobaric) $k_{m}$ of methyl caprate shows a slight downtrend

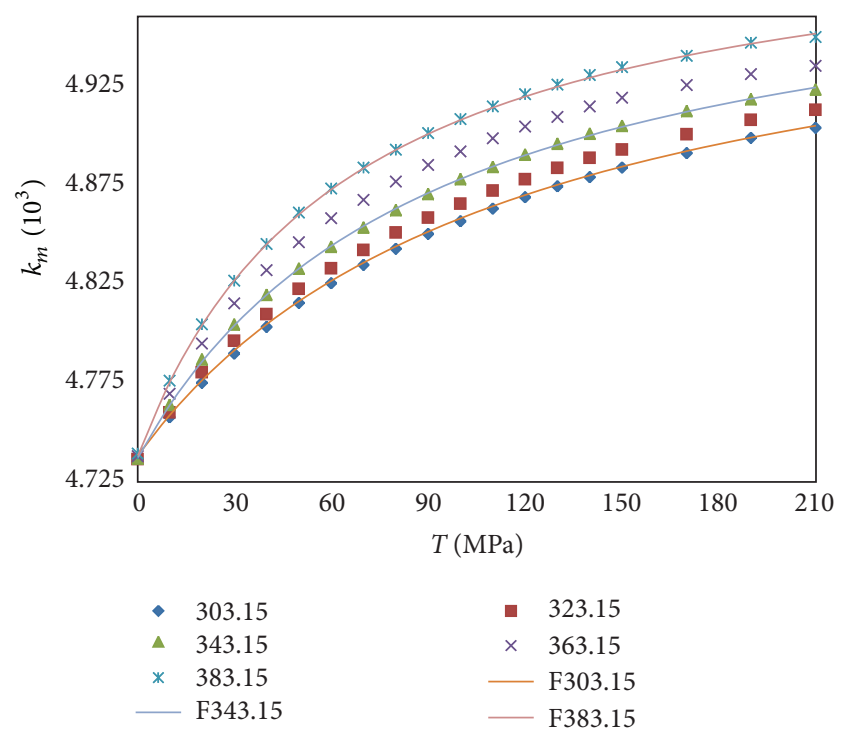

FIGURE 2: Molecular compressibility of ethyl caprate (EeC10:0) as a function of pressure along various isothermal (the lines are fitting results; the dots are calculated values).

with two-order form described in (10) but not first-order form described in Daridon's formula (5); however for $0.1 \mathrm{MPa}$ isobaric of ethyl caprate is almost horizontal; meanwhile $k_{m}$ show uptrend, which accelerates with increasing pressure, at isobaric with temperature elevated. $k_{m}$ also is dependent on temperature for ethyl and methyl caprate.

Furthermore to calculate molecular compressibility difference $k_{m}$ of ethyl caprate in isothermal line with $20 \mathrm{~K}$ step and in isobaric line with $10 \mathrm{MPa}$ step, Figures 5 and 6 can be achieved.

We can take the partial derivative with respect to pressure for (9) and to temperature for (10); thus we have

$$
\begin{gathered}
\left(\frac{\partial k_{m}}{\partial P}\right)_{T}=\frac{A_{1}}{P+A_{3}}-\frac{A_{1} P+A_{2}}{\left(P+A_{3}\right)^{2}}=\frac{A_{1} A_{3}-A_{2}}{\left(P+A_{3}\right)^{2}}, \\
\left(\frac{\partial k_{m}}{\partial T}\right)_{P}=2 B_{1} T+B_{2} .
\end{gathered}
$$


TABLE 3: Fitting coefficients for (10) along various isobaric.

\begin{tabular}{lcccccccccccc}
\hline FA ester & \multicolumn{9}{c}{ Mthyl caprate } \\
\hline $\begin{array}{l}\text { Pressure } \\
\text { Coefficients }\end{array}$ & $P 0.1$ & $P 80$ & $P 130$ & $P 150$ & $P 210$ & $P 0.1$ & $P 80$ & 130 & $P 150$ & $P 210$ \\
$\quad B_{1}$ & $-2.3 E-06$ & $1.6 E-06$ & $1.6 E-06$ & $1.6 E-06$ & $1.3 E-06$ & $2.2 E-06$ & $3.2 E-06$ & $2.6 E-06$ & $2.5 E-06$ & $2.2 E-06$ \\
$B_{2}$ & 0.00139 & -0.00056 & -0.00051 & -0.00047 & -0.00031 & -0.00094 & -0.00161 & -0.00117 & -0.00108 & -0.00094 \\
$B_{3}$ & 4.168 & 4.495 & 4.507 & 4.506 & 4.499 & 4.982 & 5.028 & 4.983 & 4.978 & 4.982 \\
Goodness of fit & & & & & & & & & & & \\
$\quad$ SSE & $2.7 E-08$ & $1.5 E-06$ & $6.5 E-07$ & $9.4 E-07$ & $1.09 E-06$ & $1.8 E-07$ & $1.9 E-07$ & $2.7 E-07$ & $3.9 E-07$ & $1.83 E-07$ \\
$\quad$-square & 0.9998 & 0.9987 & 0.9995 & 0.9993 & 0.9992 & 0.9998 & 0.9999 & 0.9998 & 0.9997 & 0.9998 \\
$\quad$ Adjusted R-square & 0.9995 & 0.9974 & 0.9991 & 0.9987 & 0.9983 & 0.9997 & 0.9997 & 0.9997 & 0.9995 & 0.9997 \\
$\quad$ RMSE & 0.00012 & 0.00088 & 0.00057 & 0.00068 & 0.00074 & 0.0003 & 0.0003 & 0.00037 & 0.00044 & 0.0003 \\
\hline
\end{tabular}

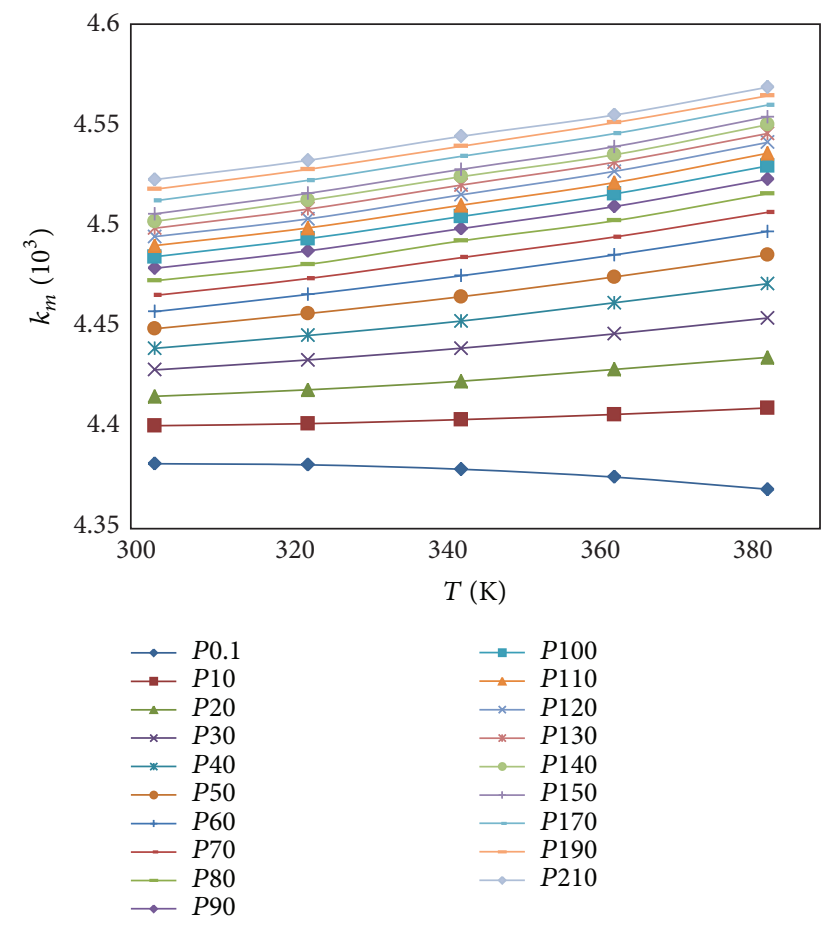

Figure 3: Molecular compressibility of methyl caprate (MeC10:0) as a function of temperature along various isobaric (only calculated values were revealed).

From Figure 5, we can find that, among various isothermals, the $\Delta k_{m} / \Delta P$ become bigger when the temperature was elevated gradually and almost keep fixed value above $60 \mathrm{MPa}$ though nearly equal at atmospheric pressure; here temperature variation plays a more important role under high pressure. Thus the $\Delta k_{m} / \Delta P$ reveal almost equality and only show dependence on temperature in high pressure, $\left(\partial k_{m} / \partial P\right)_{T} \rightarrow$ Const. The result can be achieved form the calculation of (11) coefficients; the values of $A_{1} A_{3}-A_{2}$ for various isothermal are in 23.5 to 16.2 , but $A_{3}$ is in about 110 to 65 , when pressure is large than $60 \mathrm{MPa}$ increments of $\left(P+A_{3}\right)^{2}$ acceleration.

From Figure 6, we can find that among various isobaric $\Delta k_{m} / \Delta P$ become smaller when pressure was elevated gradually and keep almost the same value above $60 \mathrm{MPa}$ with

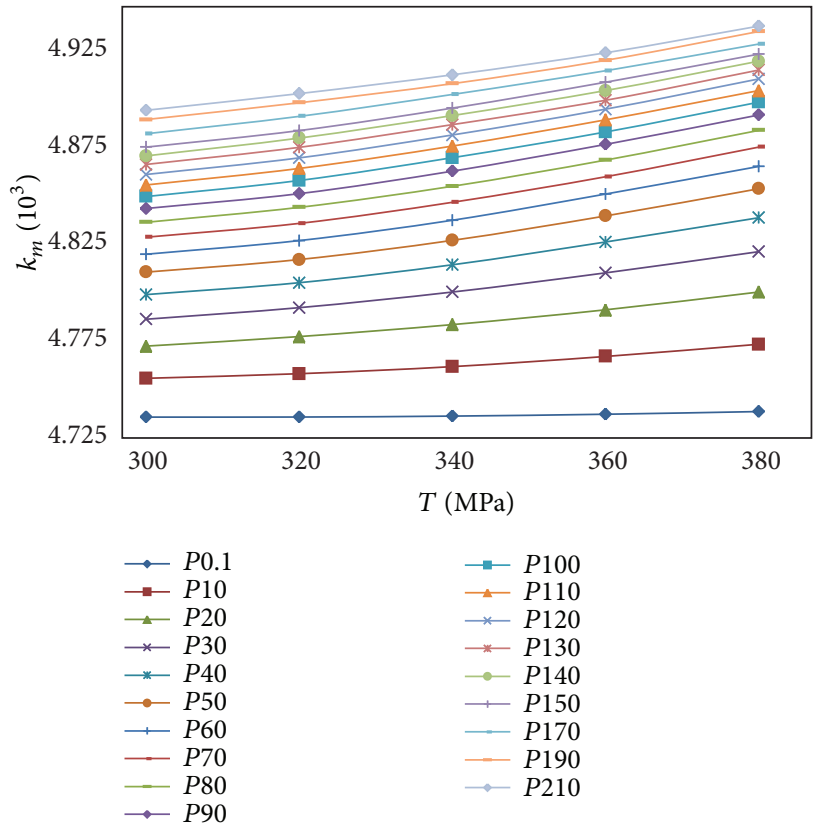

FIGURE 4: Molecular compressibility of ethyl caprate (EeC10:0) as a function of temperature along various isobaric (only calculated values were revealed).

slight dependence of pressure, That is to say the possibility of being compressed of $K_{m}$ decreases with high pressure, $\left(\partial k_{m} / \partial T\right)_{P} \simeq$ Const. Meanwhile, $\Delta k_{m} / \Delta P$ increase with elevated temperature, but the rate of increment declines rapidly based on $2 B_{1}$ which became almost the same as above $60 \mathrm{MPa}$ with $10^{-6}$ magnitude; here pressure variation reveals more contributions under low pressure.

3.2. The Data Fitting Formula of Molecular Compressibility in Three-Dimensional. According to the two-dimensional fitting analysis, a three-dimensional rational formula was introduced with the following expression:

$$
k_{m}=a+b(T+b)^{2}+\frac{c(T+b)^{2}+d}{P+e},
$$




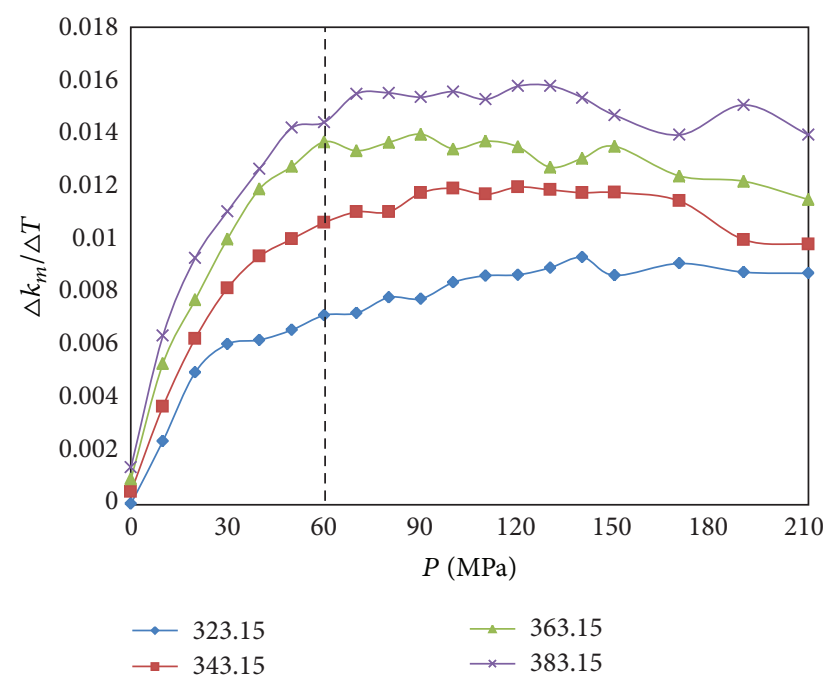

FIgure 5: The trend of differential pressure of molecular compressibility temperature along various isothermal for ethyl caprate (only calculated values were revealed).

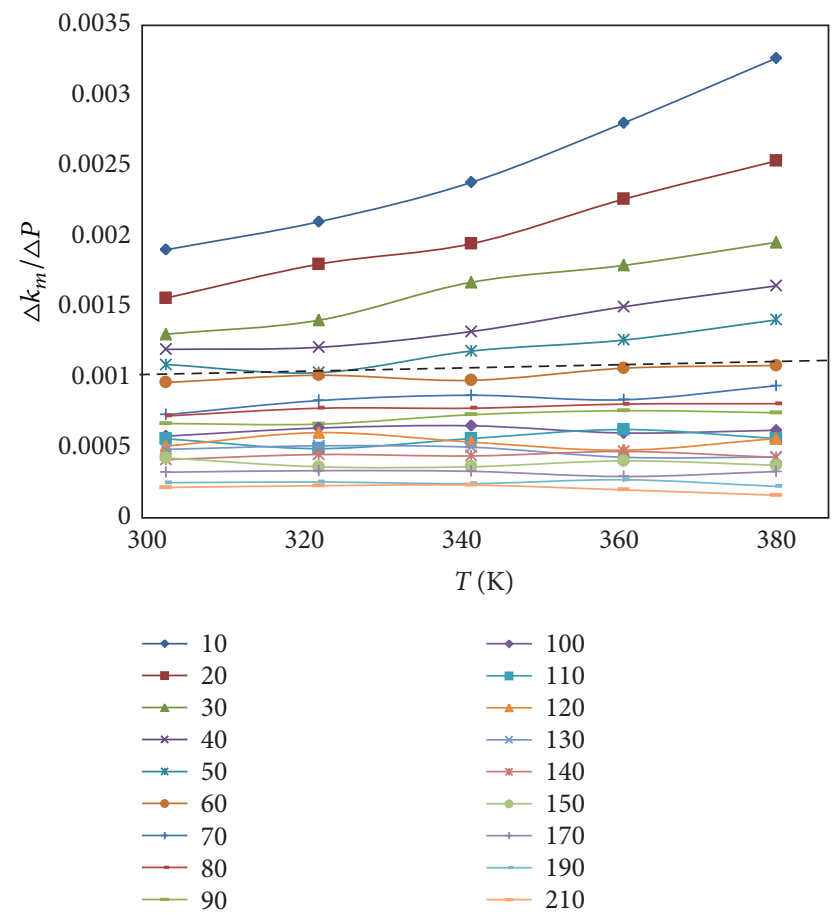

Figure 6: The trend of differential temperature ofmolecular compressibility temperature along various isobaric for ethyl caprate (only calculated values were revealed).

where $P, T$ are pressure and temperature and other fitting parameters $a, b, c, d, e$ are formula coefficients, listed in Table 4.

This formula has one-order rational form in pressure domain and two-order polynomial form in temperature domain; at the same time $\left(\partial k_{m} / \partial P\right)_{T}$ shows two-order rational form in pressure domain and $\left(\partial k_{m} / \partial T\right)_{P}$ shows one-order polynomial form in temperature. So it can be used to predict
TABLE 4: $k_{m}$ three-dimensional fitting coefficients.

\begin{tabular}{lcc}
\hline FA ester & Ethyl caprate & Methyl caprate \\
\hline Coeffiecients & & \\
$a$ & 4.451 & 4.845 \\
$b$ & $1.34 E-06$ & $1.22 E-06$ \\
$c$ & -0.0001 & $-6.91 E-05$ \\
$d$ & -6.793 & -13.2 \\
$e$ & 81.87 & 84.51 \\
& Goodness of fit & \\
SSE & 0.000307 & 0.000552 \\
$R$-square & 0.9986 & 0.9979 \\
Adjust $R$-square & 0.9986 & 0.9978 \\
RMSE & 0.001847 & 0.002476 \\
\hline
\end{tabular}

$k_{m}$ without experimental data for methyl and ethyl caprate under pressures up to $210 \mathrm{MPa}$ and temperature range from 283.15 to $403.15 \mathrm{~K}$.

3.3. The Temperature and Pressure Correction of Molecular Compressibility. Combining Figures 5 and $6, k_{m}$ shows obviously the characteristics: in lower pressure, the larger free volume will be a reasonable interpretation; in this condition the intermolecular potential mainly contributed to the pressure and the vibration of molecules is scarcely compromised; thus pressure has significant effect on it than temperature. On the contrary, in high pressure condition the molecules are much closer to the close packed state and intermolecular potential rapidly increases with tiny distance change; molecules vibration based on temperature will mainly achieve the intermolecules space and then the potential that temperature has significant effect in this time than pressure.

The Lennard-Jones potential is a mathematically simple model that approximates the interaction between a pair of neutral atoms or molecules [25]:

$$
V_{\mathrm{LJ}}=4 \varepsilon\left[\left(\frac{\sigma}{r}\right)^{12}-\left(\frac{\sigma}{r}\right)^{6}\right]=\varepsilon\left[\left(\frac{r_{m}}{r}\right)^{12}-2\left(\frac{r_{m}}{r}\right)^{6}\right],
$$

where $\varepsilon$ is the depth of the potential well, $\sigma$ is the finite distance at which the interparticle potential is zero, $r$ is the distance between the particles, and $r_{m}$ is the distance at which the potential reaches its minimum. At $r_{m}$, the potential function has the value $-\varepsilon$. However $\sigma$ is determined by temperature which represents molecular kinetic energy, and $\sigma$ increases with temperature. The repulsive force increase sharply with slight decrease of intermolecules distance.

Nevertheless, $\sigma$ was not only determined by pressure, but also implicated by the molecular configuration and size. The force situation of liquid molecules was considered in free equilibrium state under zero-pressure condition, if the gravity was neglected; here we set reference pressure $P_{\text {ref }}=$ $0.1013 \mathrm{MPa}$, and the distance between the molecules was perceived as $\sigma$ and molecules molar volume and density are set to $V_{\text {ref }}, \rho_{\text {ref }}$ under the equilibrium state. If we assume the pressure $P$ was a function of LJ-potential, $f(P)=V_{\mathrm{LJ}}$, and the space occupied by molecules was a cube, so $V_{\text {ref }}=\sigma^{3}$ and 
TABLE 5: Critical properties and parameters of ethyl caprate.

\begin{tabular}{lccccrr}
\hline Fatty ester & $T_{c}(\mathrm{~K})$ & $P_{c}(\mathrm{MPa})$ & $\omega$ & $c$ & $\varepsilon / k_{B}$ \\
\hline Ethyl caprate [18] & 690.22 & 1.869998 & 0.709 & 3.7737413 & 295.34 & $4.08 E-21$ \\
Methyl caprate [19] & 683 & 1.5701 & 0.596 & 3.2958 & 306.01 & $4.23 E-21$ \\
\hline
\end{tabular}

$V_{\text {ref }} / V_{m}=\sigma^{3} / r^{3}$. A correction coefficient $K_{P}$ of pressure was introduced and the Lennard-Jones potential can be written as

$$
\begin{aligned}
K_{P} & =f(P)=\frac{V_{\mathrm{LJ}}}{\varepsilon}=4\left[\left(\frac{V_{\text {ref }}}{V_{m}}\right)^{4}-\left(\frac{V_{\text {ref }}}{V_{m}}\right)^{2}\right] \\
& =4\left[\left(\frac{\rho}{\rho_{\text {ref }}}\right)^{4}-\left(\frac{\rho}{\rho_{\text {ref }}}\right)^{2}\right] .
\end{aligned}
$$

For temperature correction of $k_{m}$, energy equipartition theorem [26], which makes quantitative predictions, was considered, and it gave the total average kinetic and potential energies for a system at a given temperature in thermal equilibrium. The oscillating molecules have average energy

$$
\langle H\rangle=\left\langle H_{\text {kinetic }}\right\rangle+\left\langle H_{\text {potential }}\right\rangle=\frac{1}{2} k_{B} T+\frac{1}{2} k_{B} T=k_{B} T,
$$

where the angular brackets $\langle\cdots\rangle$ denote the average of the enclosed quantity. So $\left(\varepsilon / k_{B} T-\varepsilon / k_{B} T_{\text {ref }}\right)$ will be a correction factor to charge temperature effect on $k_{m}$

$$
K_{T}=\operatorname{Exp}\left(\frac{\varepsilon}{k_{B} T_{\text {ref }}}-\frac{\varepsilon}{k_{B} T}\right)
$$

where $\varepsilon$ is the depth of the potential well and also represents a unit of energy. $T_{\text {ref }}$, which is reference temperature, was considered as the cloud point temperature of an organic liquid under reference pressure $P_{\text {ref }}$, for ethyl caprate $T_{\text {ref }}$ being $253.15 \mathrm{~K}$ and for methyl caprate $T_{\text {ref }}$ being $260.15 \mathrm{~K}$.

The Elliott, Suresh, and Donohue (ESD) equation of state accounts for the effect of the shape of a nonpolar molecule and can be extended to polymers with the addition of an extra term. The EOS itself was developed through modeling computer simulations and should capture the essential physics of the size, shape, and hydrogen bonding [27].

In ESD, the shape factor $c=1+3.535 \omega+0.533 \omega^{2}$, where $\omega$ is the acentric factor. And the following equation shows the energy factor $\varepsilon / k_{B}$ is related to the shape parameter $c$ :

$$
\frac{\varepsilon}{k_{B}}=T_{c} \frac{1+0.945(c-1)+0.134(c-1)^{2}}{1.023+2.225(c-1)+0.478(c-1)^{2}}
$$

In this case, these calculation methods of relevant parameters follow EOS, and the calculation data were cited from $[18,19]$ and listed in Table 5 with the calculated parameters value.

By data calculation and comparison, the pressure correction factor is written as $K_{P}^{3 / 4}$ and the final correction formula of molecular compressibility are written as

$$
k_{m}=k_{m \mathrm{ref}}\left(1+\frac{0.15\left(0.1 K_{P}\right)^{3 / 4}}{K_{T}^{1 / 7}}\right),
$$

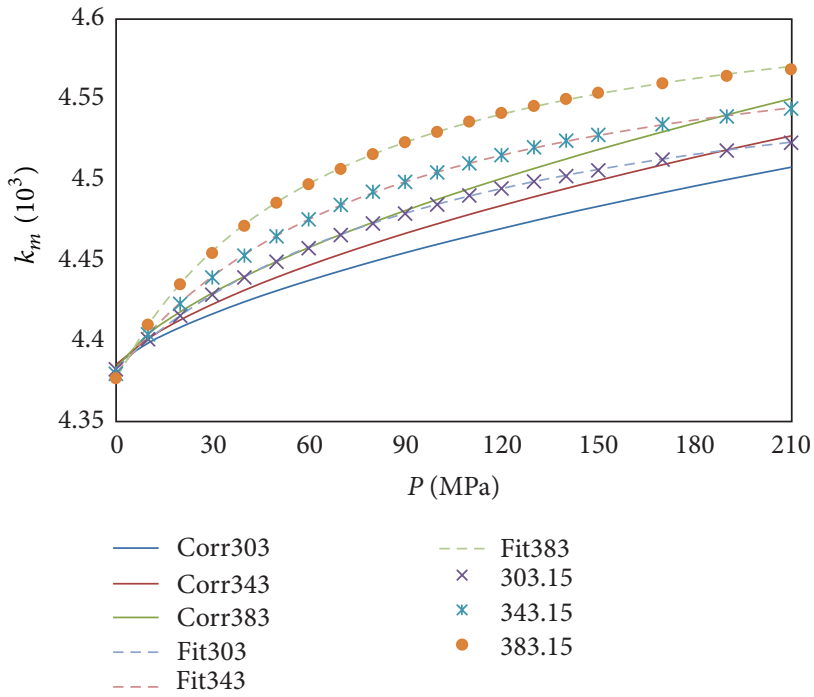

FIGURE 7: Molecular compressibility's values calculated from three formulas for methyl caprate along various isothermals (dots for (8), dotted lines for (13), and lines for (19)).

where the reference molecular compressibility, $k_{m \text { ref }}$, is obtained from the additivity of the subgroup of molecule under reference pressure and temperature and listed in Table 1 . So if the density under specific pressure and temperature was obtained, the molecular compressibility could be calculated by (19). Because sound velocity vanishes from calculation, molecular compressibility can be obtained more conveniently.

3.4. The Accuracy Comparison among Molecular Compressibility Expressions. To apply (8), (13), and (19), the requirements of experimental data are different. Formula (8) needs density and sound velocity data, (19) only need density data, and (13) does not need experimental data. Further (8) is connected to dynamic precess, and (19) reflects statistical state value, and (13) derives from calculated results of (8). The change of density is more direct property associated to compressibility than sound velocity, so (19) should be more accurate. The results comparison of three formulas was showed in Figures 7 and 8 for methyl caprate and ethyl caprate, respectively. Formula (19) reveal bigger difference at whole $P-T$ domain.

To evaluate the predictive capacity of the formulas aforementioned, the relative deviations (RDs) between two formulas of $k_{m}$ were estimated according to

$$
\mathrm{RD}(\%)=\frac{k_{m \text { formula }-i}-k_{m f \text { ormula }-j}}{k_{\text {mformula- } j}} \% .
$$




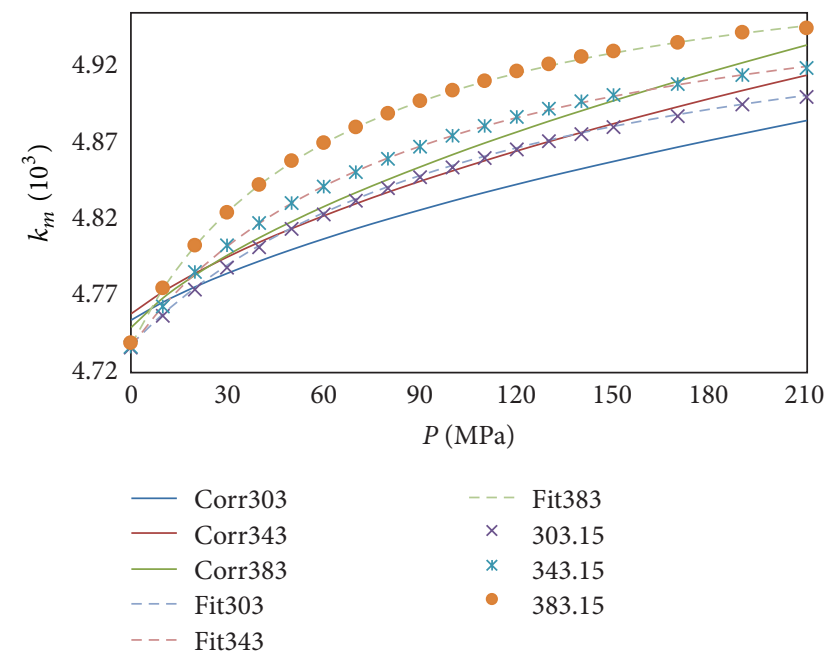

FIGURE 8: Molecular compressibility's values calculated from three formulas for ethyl caprate along various isothermals (dots for (8), dotted lines for (13), and lines for (19)).

TABLE 6: OARD \% of calculated results for three methods under 303 to $383 \mathrm{~K}, 0.1$ to $210 \mathrm{MPa}$.

\begin{tabular}{lcc}
\hline & Methyl caprate & Ethyl caprate \\
\hline Formula (8) versus (13) & -0.00818 & -0.00631 \\
Formula (8) versus (19) & 0.530178 & 0.380222 \\
Formula (13) versus (19) & 0.538393 & 0.386536 \\
\hline
\end{tabular}

The average relative deviation (ARD) was calculated as a summation of RD over $N_{p}$ calculated data points at a certain temperature. The overall average relative deviation (OARD) was calculated by (21), where $N_{s}$ is the number of calculated points:

$$
\operatorname{OARD}(\%)=\frac{\sum_{n} \mathrm{ARD}}{N_{s}}
$$

An error comparison of calculation and prediction results among (8), (13), and (19) was presented in Table 6 for two FA esters. In the high pressure and temperature range of experimental data have an uncertainty (about $0.3 \%$ or more in $\mathrm{AAD} \%$ ) that increases uncertainty of the comparison. The OARD\% information also indicates that the formulas do not introduce systematic errors. The comparison results of these methods, which were derived from different principle, show clearly the reliability of these methods when applied in extrapolation and confirm their purely predictive character. Because the molecular compressibility data is scarce under high pressure and higher temperature, it is difficult to determine which one has better performance under extended pressure and temperature ranges.

More recently, Cunha et al. [20] proposed a new atomic contribution model to predict speed of sound, the group contribution model, which was listed in Table 1 and calculated by (5) and was replaced by seven atomic compounds: $C_{p a r}$ (for paraffinic carbon), $\mathrm{C}_{\mathrm{Olef}}$ (for olefinic carbon), $\mathrm{C}_{\text {Arom }}$ (for aromatic carbon), $\mathrm{H}$ (for hydrogen), $\mathrm{O}_{\mathrm{sp} 3}$ (for ether oxygen),

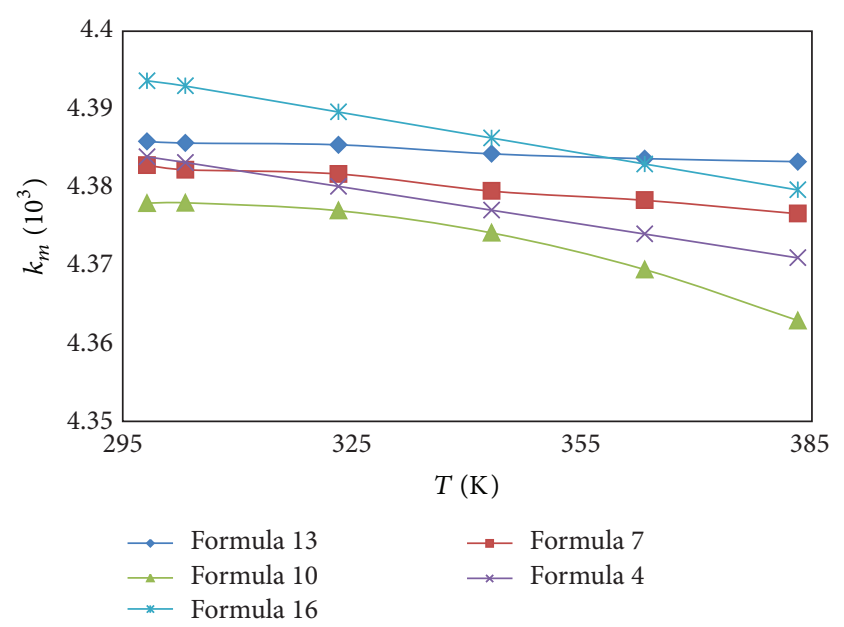

FIGURE 9: Molecular compressibility's values calculated from five formulas for methyl caprate at atmospheric pressure.

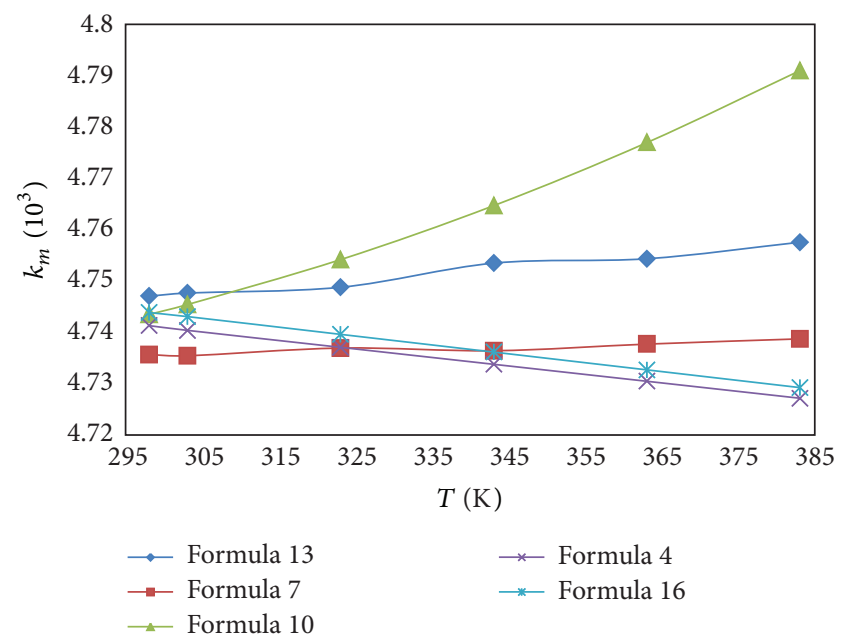

FIGURE 10: Molecular compressibility's values calculated from five formulas for ethyl caprate at atmospheric pressure.

$\mathrm{O}_{\mathrm{sp} 2}$ (for ester group, being the $\mathrm{COO}$ decomposed into one $\mathrm{C}_{\mathrm{Olef}}$, one $\mathrm{O}_{\mathrm{sp2}}$, and one $\mathrm{O}_{\mathrm{sp} 3}$ ), and $\mathrm{O}_{\mathrm{Alc}}$ (for alcohol oxygen). $\chi_{a}$ is the atom linear temperature correction parameter and $a$ runs over all atoms; however $\chi_{a}$ have different values only for $\mathrm{O}_{\mathrm{Alc}}$ and $\mathrm{O}_{\mathrm{sp} 2}$. Adjusted Wada's constant contributions are presented in Table 7, and Wada's constant was calculated by

$$
k_{m}(T)=\sum_{\alpha=1}^{n} N_{\alpha} k_{m \alpha}\left(1-\chi_{\alpha}(T-298.15)\right)
$$

but the two methods are available only at atmospheric pressure, so we take $0.1013 \mathrm{MPa}$ isobaric value to compare the accuracy of five methods; the comparison results for methyl and ethyl caprate were listed in Figures 9 and 10.

The values of (8) are obtained from the experimental speed of sound and density data, so (5) and (19) have higher consistency at atmospheric pressure. Formula (13) showed bigger difference, while (5) and (22) for ethyl caprate at $0.1 \mathrm{MPa}$ isobaric. 
TABLE 7: Atomic contribution values and temperature correction coefficients [20].

\begin{tabular}{lccccccc}
\hline & $\mathrm{C}_{\mathrm{Par}}$ & $\mathrm{C}_{\text {Olef }}$ & $\mathrm{C}_{\text {Arom }}$ & $\mathrm{H}$ & $\mathrm{O}_{\text {sp3 }}$ & $\mathrm{O}_{\text {Alc }}$ & $\mathrm{O}_{\text {sp2 }}$ \\
\hline $10^{3}$ Wada's constant (SI) & 0.0113 & 0.1234 & 0.1348 & 0.1694 & 0.1738 & 0.094 & 0.2568 \\
$10^{3} \chi\left(\mathrm{K}^{-1}\right)$ & 0.0191 & 0.0191 & 0.0191 & 0.0191 & 0.0191 & -1.3064 & 0.3488 \\
\hline
\end{tabular}

\section{Conclusion}

The molecular compressibility that was considered as a constant is challenged as a function of pressure and temperature. By data fitting a first order rational function in pressure domain and a two-order polynomial function in temperature domain were achieved. The results of $2 \mathrm{D}$ data fitting showed that molecular compressibility has an evident dependence of temperature and pressure. Partial derivative of pressure and temperature was introduced to analyze this dependency further. The 3D fitting results of molecular compressibility obviously showed that pressure reveals more contributions under low pressure, while temperature plays a more important role under high pressure.

This dependence was correlated with molecular potential energy and kinetic energy by developing pressure and temperature correction coefficients, which are derived from Lennard-Jones potential function and energy equipartition theorem. Combining the two correction coefficients, the new molecular compressibility predicting formula is achieved. As a result, the molecular compressibility at working conditions of injection system can be calculated directly by density data in corresponding condition. With the maximum $0.5384 \%$ OARD, the consistency of calculation results demonstrated the validity of three expressions and the pressure and temperature dependence of molecular compressibility.

The correction expression (Formula (19)), which contains "shape factor" of molecules and only requires density data, has conceivable prospects to be extended to other FA esters and biodiesels. But (19) needs more mathematical way to improve its accuracy; the HILO-based error correction scheme used to improve Gaussian mixture models (GMMs) [28] would be an idea worthy of learning.

\section{Conflict of Interests}

The authors declare that there is no conflict of interests regarding the publication of this paper.

\section{References}

[1] F. Boudy and P. Seers, "Impact of physical properties of biodiesel on the injection process in a common-rail direct injection system," Energy Conversion and Management, vol. 50, no. 12, pp. 2905-2912, 2009.

[2] A. L. Boehman, D. Morris, J. Szybist, and E. Esen, "The impact of the bulk modulus of diesel fuels on fuel injection timing," Energy \& Fuels, vol. 18, no. 6, pp. 1877-1882, 2004.

[3] M. E. Tat and J. H. van Gerpen, "Measurement of biodiesel sound velocity and its impact on injection timing," Final Report for National Renewable Energy Laboratory ACG-8-18066-11, 2000 .
[4] G. Knothe, “'Designer' biodiesel: optimizing fatty ester composition to improve fuel properties," Energy \& Fuels, vol. 22, no. 2, pp. 1358-1364, 2008.

[5] G. Knothe, C. A. Sharp, and T. W. Ryan III, "Exhaust emissions of biodiesel, petrodiesel, neat methyl esters, and alkanes in a new technology engine," Energy \& Fuels, vol. 20, no. 1, pp. 403408, 2006.

[6] R. Payri, F. J. Salvador, J. Gimeno, and G. Bracho, "The effect of temperature and pressure on thermodynamic properties of diesel and biodiesel fuels," Fuel, vol. 90, no. 3, pp. 1172-1180, 2011.

[7] M. R. Rao, "The adiabatic compressibility of liquids," The Journal of Chemical Physics, vol. 14, p. 699, 1946.

[8] Y. Wada, "On the relation between compressibility and molal volume of organic liquids," Journal of the Physical Society of Japan, vol. 4, no. 4-6, pp. 280-283, 1949.

[9] W. Schaaffs, "Schllgeschwindigkeit und Molekülstuktur in Flüssigkeiten," Zeitschrift für Physikalische Chemie, vol. 196, p. 413, 1951.

[10] W. Schaaffs, "Der ultraschall und die struktur der flüssigkeiten," Il Nuovo Cimento, vol. 7, no. 2, supplement, pp. 286-295, 1950.

[11] W. Schaaffs, "Die Additivitiätsgesgtze der schllgeschwindigkeit in flüssigkeiten," Ergebnisse der Exakten Naturwiss, vol. 25, p. 109, 1951.

[12] J. L. Daridon, J. A. P. Coutinho, E. H. I. Ndiaye, and M. L. L. Paredes, "Novel data and a group contribution method for the prediction of the sound velocity and isentropic Compressibility of pure fatty acids methyl and ethyl esters," Fuel, vol. 105, pp. 466-470, 2013.

[13] S. V. D. Freitas, D. L. Cunha, R. A. Reis et al., "Application of wada's group contribution method to the prediction of the sound velocity of biodiesel," Energy \& Fuels, vol. 27, no. 3, pp. 1365-1370, 2013.

[14] S. V. D. Freitas, A. Santos, M. L. C. J. Moita et al., "Measurement and prediction of speeds of sound of fatty acid ethyl esters and ethylic biodiesels," Fuel, vol. 108, pp. 840-845, 2013.

[15] E. H. I. Ndiaye, D. Nasri, and J. L. Daridon, "Sound velocity, density, and derivative properties of fatty acid methyl and ethyl esters under high pressure: methyl caprate and ethyl caprate," Journal of Chemical \& Engineering Data, vol. 57, no. 10, pp. 26672676, 2012.

[16] S. V. D. Freitas, M. L. L. Paredes, J. L. Daridon, A. S. Lima, and J. A. P. Coutinho, "Measurement and prediction of the speed of sound of biodiesel fuels," Fuel, vol. 103, pp. 1018-1022, 2013.

[17] F. Shi and J. Chen, "Influence of injection temperature on atomization characteristics of biodiesel," Transactions of the Chinese Society of Agricultural Machinery, vol. 44, no. 7, pp. 3338, 2013.

[18] M. C. Costa, L. A. D. Boros, M. L. S. Batista, J. A. P. Coutinho, M. A. Kraenbuhl, and A. J. A. Meirelles, "Phase diagrams of mixtures of ethyl palmitate with fatty acid ethyl esters," Fuel, vol. 91, no. 1, pp. 177-181, 2012.

[19] O. C. Didz, Measurement and Modelling Methodology for Heavy Oil and Bitumen Vapour Pressure, University of Calgary, 2012. 
[20] D. L. Cunha, J. A. P. Coutinho, R. A. Reis, and M. L. L. Paredes, "An atomic contribution model for the prediction of speed of sound," Fluid Phase Equilibria, vol. 358, pp. 108-113, 2013.

[21] M. E. Tat, J. H. Van Gerpen, S. Soylu, M. Canakci, A. Monyem, and S. Wormley, "The sound velocity and isentropic bulk modulus of biodiesel at $21^{\circ} \mathrm{C}$ from atmospheric pressure to 35 MPa," Journal of the American Oil Chemists' Society, vol. 77, no. 3, pp. 285-289, 2000.

[22] M. E. Tat and J. H. van Gerpen, "Speed of sound and isentropic bulk modulus of alkyl monoesters at elevated temperatures and pressures," Journal of the American Oil Chemists' Society, vol. 80, no. 12, pp. 1249-1256, 2003.

[23] L. A. Davis and R. B. Gordon, "Compression of mercury at high pressure," The Journal of Chemical Physics, vol. 46, no. 7, pp. 2650-2660, 1967.

[24] J. L. Daridon, B. Lagourette, and J. P. Grolier, "Experimental measurements of the speed of sound in $n$-Hexane from 293 to $373 \mathrm{~K}$ and up to $150 \mathrm{MPa}$," International Journal of Thermophysics, vol. 19, no. 1, pp. 145-160, 1998.

[25] J. E. Lennard-Jones, "On the determination of molecular fields. II.From the equation of state of a gas," Proceedings of the Royal Society of London A, vol. 106, no. 738, pp. 463-477, 1924.

[26] K. Huang, Statistical Mechanics, John Wiley \& Sons, 2nd edition, 1987.

[27] J. R. Elliott Jr., S. J. Suresh, and M. D. Donohue, "A simple equation of state for nonspherical and associating molecules," Industrial \& Engineering Chemistry Research, vol. 29, no. 7, pp. 1476-1485, 1990.

[28] J. H. Park and H. K. Kim, "Dual-microphone voice activity detection incorporating gaussian mixture models with an error correction scheme in non-stationary noise environments," International Journal of Innovative Computing, Information and Control, vol. 9, no. 6, pp. 2533-2542, 2013. 


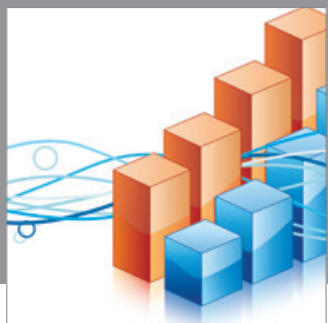

Advances in

Operations Research

mansans

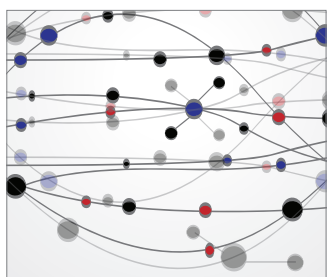

The Scientific World Journal
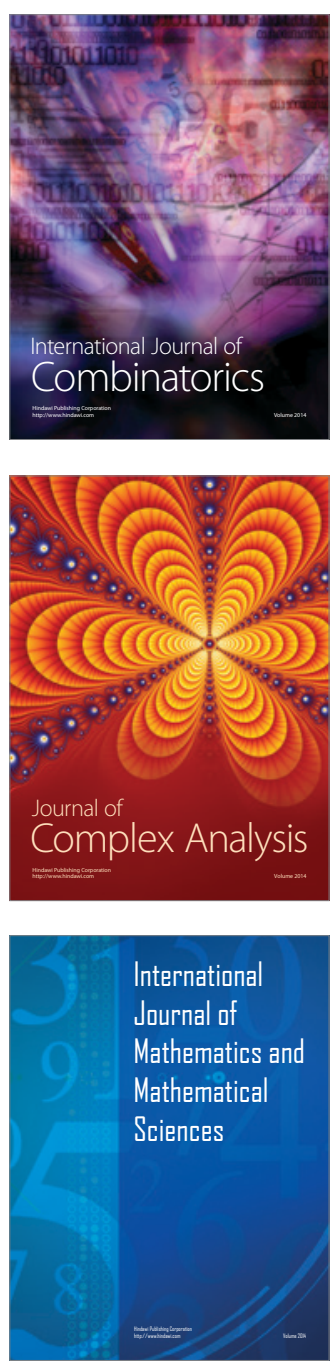
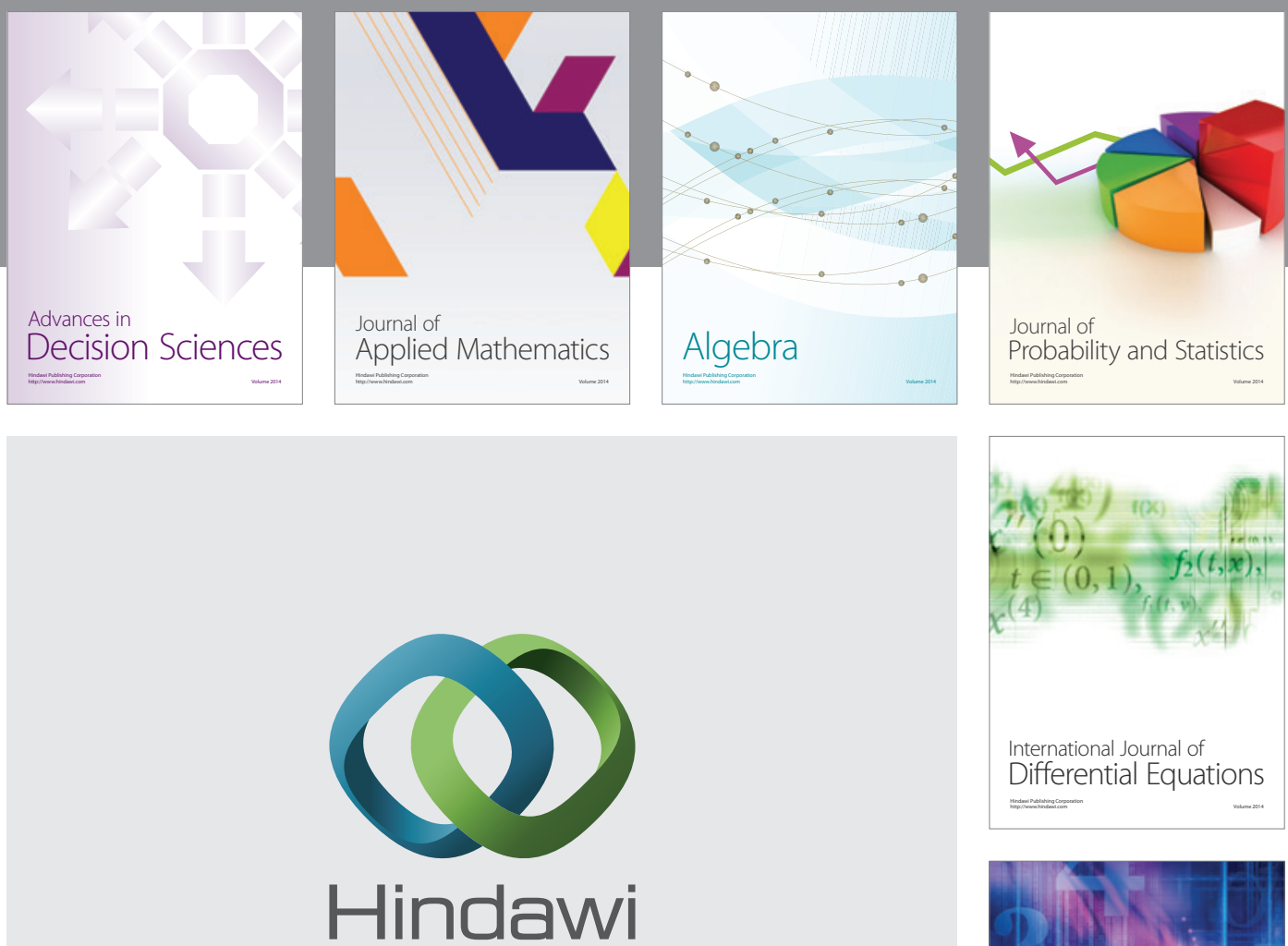

Submit your manuscripts at http://www.hindawi.com
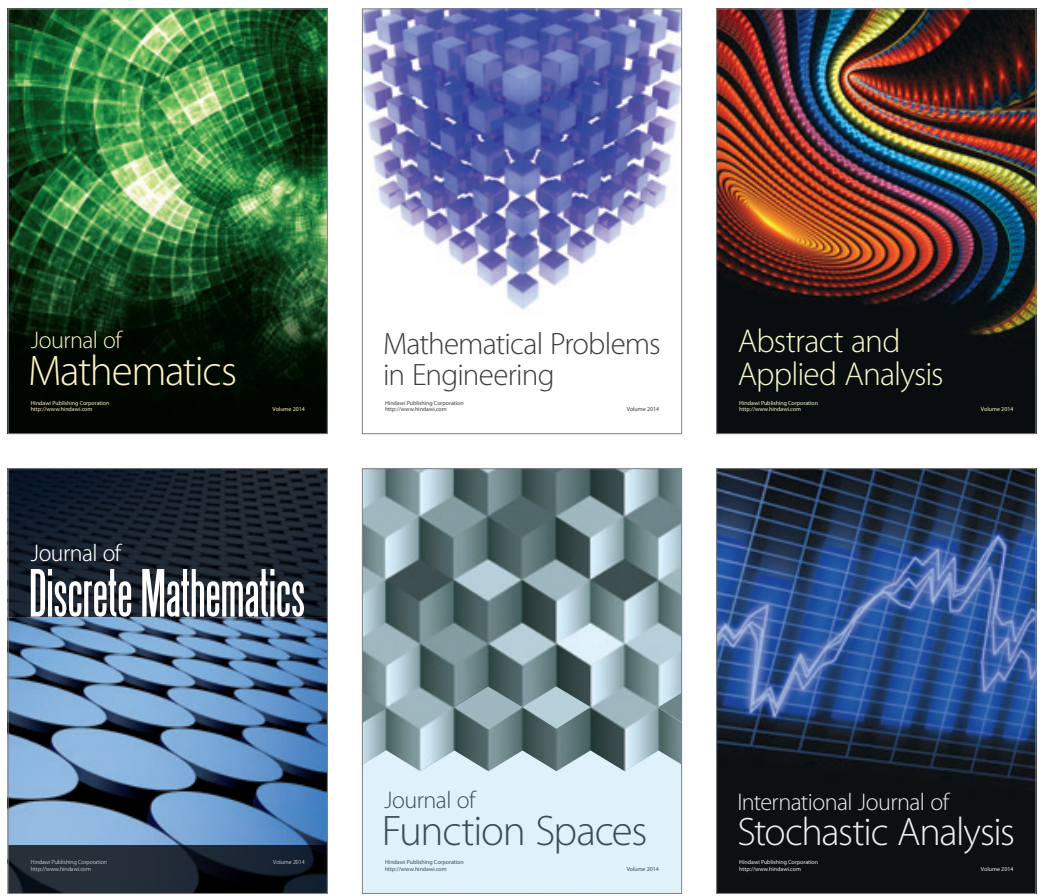

Journal of

Function Spaces

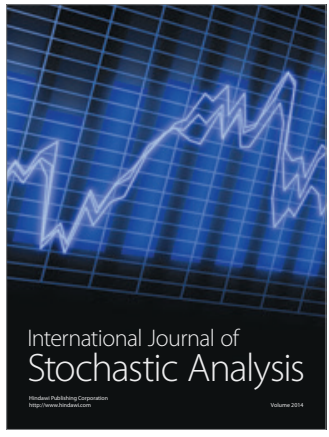

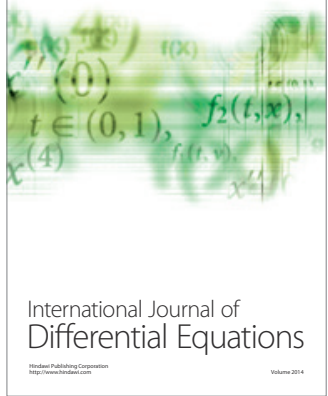
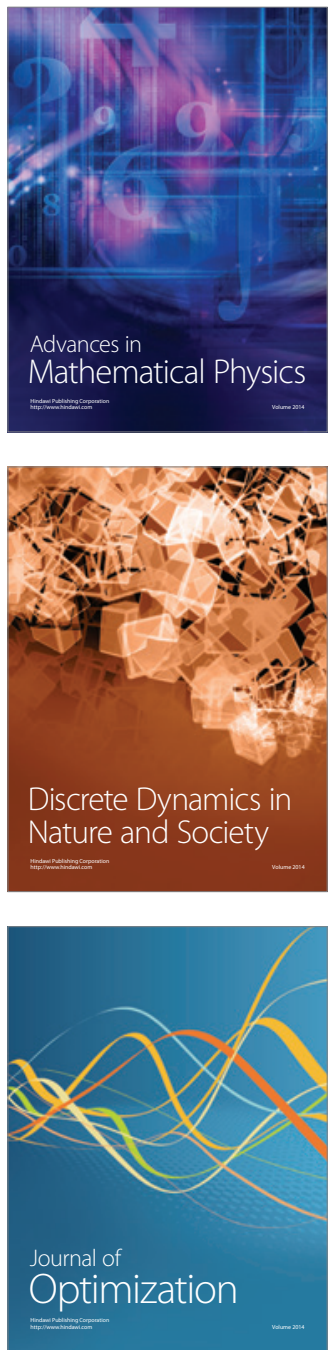